\title{
Chronic fatigue syndrome with history of severe infection combined altered blood oxidant status, and reduced potassium efflux and muscle excitability at exercise
}

\author{
Yves Jammes $^{1,2 *}$, Jean Guillaume Steinberg ${ }^{2}$, Regis Guieu ${ }^{1}$, Stephane Delliaux ${ }^{1,2}$ \\ ${ }^{1}$ UMR MD2, Faculty of Medicine, Aix-Marseille University, Marseille, France \\ ${ }^{2}$ Clinical Respiratory Physiology, Exercise Testing Laboratory, North Hospital, Public Health-Hospitals in Marseille, Marseille, France \\ Email: *Yves.jammes@univ-amu.fr
}

Received 30 January 2013; revised 28 February 2013; accepted 14 March 2013

Copyright (C) 2013 Yves Jammes et al. This is an open access article distributed under the Creative Commons Attribution License, which permits unrestricted use, distribution, and reproduction in any medium, provided the original work is properly cited.

\begin{abstract}
It is documented that chronic fatigue syndrome (CFS) combines enhanced oxidative stress with altered muscle excitability. We hypothesized that these disorders may be accentuated when severe infection preceded the CFS symptoms. This case-control study compared 55 CFS patients to a matched control group of 40 healthy subjects. In twenty-five CFS patients, severe infection was reported within the three to seven month period preceding the CFS symptoms. The others had practiced sport at high level. Plasma concentrations of potassium, a marker of lipid peroxidation (thiobarbituric acid reactive substances, TBARS), and an endogenous antioxidant (reduced ascorbic acid, RAA) were measured. Action potential (M-wave) was evoked in the vastus lateralis muscle to explore the muscle membrane excitability. All subjects performed a maximal incremental cycling exercise. Compared to control subjects, all CFS patients presented an elevated resting TBARS level and, during and after exercise, an altered M-wave configuration. History of infection was associated with marked significant increase in resting TBARS level, enhanced $M$-wave alterations, and also reduced exercise-induced potassium efflux. The magnitude of exercise-induced M-wave alterations was proportional to the baseline TBARS level. Severe infection preceding CFS seems to constitute a stressor inducing altered blood oxidant status and a reduced muscle excitability at work.
\end{abstract}

Keywords: Chronic Fatigue Syndrome; Infectious Diseases; Muscle Excitability; Oxidative Stress

\footnotetext{
"Corresponding author.
}

\section{INTRODUCTION}

The Chronic Fatigue Syndrome (CFS) results in a severe impairment in sports and daily activities [1]. Exerciseinduced CFS pathology is suspected [2,3]. However, CFS often follows severe bacterial or viral infection [4-6] and various suspected pathogens, like Epstein-Barr virus, cytomegalovirus, human herpes virus, enterovirus, parvovirus and mycoplasma, have been considered. Previous studies have reported marked alterations in biological and physiological responses to maximal exercise in CFS patients compared to healthy sedentary subjects [7-12]. These differences included marked post-exercise alterations of the muscle membrane excitability $[7,8]$, an early and lengthened exercise-induced oxidative stress [7-13], and an attenuated heat shock proteins response (HSP) $[8,13]$.

An excessive production of reactive oxygen species (ROS), responsible for membrane lipid peroxidation [14], exerts well known effects on isolated human and animal tissues, including the loss of membrane excitability due to an altered activation of potassium $\left(\mathrm{K}^{+}\right)$channels in cardiac myocytes [15] and skeletal muscle fibres [16]. In healthy humans, muscle biopsies have demonstrated that the strength of membrane excitation is proportional to the $\mathrm{K}^{+}$efflux [17], detectable in plasma [18]. An increased ROS production exerts an inhibitory action on the $\mathrm{Na}^{+}-\mathrm{K}^{+}$pump activity [14], reducing the potassium outflow and the muscle membrane excitability. The CFS study by Fulle et al. [19] has confirmed a deregulation of the $\mathrm{Na}^{+} / \mathrm{K}^{+}$and $\mathrm{Ca}^{2+}$-ATPase pumps and the alterations of ryanodine channels in the sarcoplasmic reticulum membranes. These authors [19] have hypothesized that the deregulation of pump activities could result from an increased fluidity of the sarcoplasmic membrane due to the ROS-induced formation of lipid hydroperoxides. It is 
well documented that severe bacterial [20] and viral [2125] infections, including the H1N1 influenza [24,25], induce an oxidative stress. Thus, infection could be responsible for an altered membrane excitability due to reduced $\mathrm{K}^{+}$exchanges through the muscle membrane.

Recording the compound evoked muscle action potentials (M-wave) with surface electrodes is a non-invasive tool, commonly used to explore the muscle membrane excitability in resting and exercising humans [7,26,27]. An impaired excitation of the muscle fibres is assessed when the M-wave declines and becomes broader. In healthy sedentary subjects, modest changes in M-wave duration and amplitude occur during and after a maximal incremental cycling exercise [7,26,27]. On the opposite, CFS patients present a significant lengthening of the neuromuscular conduction time (CT), a reduction of the M-wave amplitude, and lengthened M-wave duration, the altered muscle excitability often persisting at the end of a 30 -min recovery period [7].

We hypothesized that the disorders of blood oxidant status and altered muscle membrane excitability could be accentuated in CFS patients with history of severe infection.

\section{METHODS}

\subsection{Subjects}

Fifty five CFS patients were explored. The criteria to include them were 1) clinical [1]: persistent or relapsing fatigue for at least 6 consecutive months or longer and four or more of the following symptoms: post-exertional malaise, impaired memory or concentration, unrefreshing sleep, muscle pain, multi-joint pain, tender cervical or axillary lymph nodes, sore throat, headache; and 2) bio- logical: the combination of exercise-induced alteration of the muscle membrane excitability $[7,8]$ and an early and lengthened exercise-induced oxidative stress [7-13]. Depression patients and chronic disease patients were excluded in this study. Retrospectively, two groups of CFS patients were identified considering their history of sport practice and severe acute infection. Thirty of them had practiced sport at high level ( $>6 \mathrm{~h}$ per week) for more than six years before the symptoms had occurred and no history of severe infection was reported by their practitioners. In twenty five patients, who did not regularly practice any sport, severe infection (H1N1 influenza: 5; pneumonia: 7; encephalomyelitis: 6; sepsis: 4; unidentified: 3) was reported within the three to seven month period preceding the onset of CFS symptoms. The two groups of CFS patients were called NI (not infected) and I (infected), respectively. The data were compared to those obtained in a gender, age-, and weight-matched control group of forty Caucasian healthy volunteers who consulted for a medical check up and were coming from the same type of socioeconomic class. Additional biochemical analyses in venous blood samples were approved by the Ethics Committee of our institution (CCPPRB Marseille 1) and the protocol was performed in accordance with the Declaration of Helsinki. The procedures were carried out with the adequate understanding and written consent of the subjects. The morphological characteristics of NI and I CFS patients and healthy subjects are collected in Table $\mathbf{1}$.

\subsection{Physiological Measurements}

The functional examinations at rest consisted in ECG recording, measurements of the arterial blood pressure

Table 1. Comparison of controls and CFS patients. Morphological characteristics, maximal performances at work, baseline and maximal variations of venous plasma concentrations of lactic acid (LA), potassium $\left(\mathrm{K}^{+}\right)$, and markers of oxidant/antioxidant status (TBARS, RAA) in healthy (control) subjects and CFS patients having history or not of recent severe infection (Infected or I CFS, non infected or NI CFS).

\begin{tabular}{|c|c|c|c|}
\hline & Controls & NI CFS group & I CFS group \\
\hline $\mathrm{N}$ & 40 & 30 & 25 \\
\hline $\mathrm{F} / \mathrm{M}$ ratio & $14 / 26$ & $10 / 20$ & $12 / 13$ \\
\hline Age, $y$ & $44 \pm 5$ & $39 \pm 3$ & $40 \pm 3$ \\
\hline Weight, kg & $71 \pm 4$ & $71 \pm 2$ & $66 \pm 2$ \\
\hline $\mathrm{VO}_{2} \max , \operatorname{mlSTPD} \cdot \min ^{-1} \cdot \mathrm{kg}^{-1}$ & $31 \pm 3$ & $29 \pm 2$ & $27 \pm 1$ \\
\hline LA rest, $\mathrm{mmol} \cdot \mathrm{l}^{-1}$ & $1.30 \pm 0.16$ & $1.43 \pm 0.11$ & $1.40 \pm 0.10$ \\
\hline$\Delta$ LAmax, $\%$ rest & $+382 \pm 21$ & $+381 \pm 51$ & $+328 \pm 43$ \\
\hline $\mathrm{K}^{+}$rest, $\mathrm{mmol} \cdot \mathrm{l}^{-1}$ & $4.02 \pm 0.04$ & $4.00 \pm 0.05$ & $3.93 \pm 0.06$ \\
\hline$\Delta \mathrm{K}^{+} \max , \%$ rest & $+29 \pm 3$ & $+25 \pm 2^{*}$ & $+17 \pm 4^{\#}$ \\
\hline TBARS rest, $\mathrm{nmol}^{\prime} \mathrm{ml}^{-1 \#}$ & $1.36 \pm 0.07$ & $1.87 \pm 0.21^{\#, * * *}$ & $3.48 \pm 0.40^{\#}$ \\
\hline$\Delta$ TBARSmax, $\%$ rest & $+57 \pm 11$ & $+149 \pm 25^{\#, * *}$ & $+66 \pm 18$ \\
\hline $\mathrm{RAA}$, rest $\mathrm{nmol} \cdot \mathrm{ml}^{-1}$ & $148 \pm 10$ & $134 \pm 15$ & $157 \pm 19$ \\
\hline$\Delta$ RAAmax, $\%$ rest & $-51 \pm 8$ & $-30 \pm 5$ & $-33 \pm 7$ \\
\hline
\end{tabular}

Values are mean \pm SE. Symbol $\#$ indicates significant difference between controls and CFS patients $(*: p<0.05)$ and asterisk depicts intergroup CFS differences $\left(: \mathrm{p}<0.05 ;{ }^{* *}: \mathrm{p}<0.01 ;{ }^{* * *}: \mathrm{p}<0.001\right)$. 
measurements using a sphygmomanometer, the percutaneous measurement of oxygen saturation $\left(\mathrm{SpO}_{2}\right)$ using an infrared analyser (Nellcor model N3000, Tx, USA), and ventilation and respiratory gas exchange. A face mask was designed to form an air-tight seal over the patient's nose and mouth, with all the inspired and expired gas going into a turbine flowmeter (Triple $\mathrm{V}$ digital volume transducer, Jaeger, Germany) giving measurements of minute ventilation. Fast-response paramagnetic $\mathrm{O}_{2}$ and infrared $\mathrm{CO}_{2}$ analyzers measured the end-tidal partial pressures of $\mathrm{O}_{2}$ and $\mathrm{CO}_{2}$, respectively. Exercise trials were performed on an electrically braked cycle ergometer (Ergometrics ER 800, Jaeger, Bunnik, The Netherlands) connected to a microcomputer software (Oxycon beta, Jaeger, Bunnik, The Netherlands). The exercise bout was preceded by a $2-$ min $0-\mathrm{W}$ pedalling period then the load was increased as a ramp $(20 \mathrm{~W} / \mathrm{min})$ until the peak $\mathrm{O}_{2}$ uptake $\left(\mathrm{VO}_{2} \mathrm{max}\right)$ was measured. The criteria to establish $\mathrm{VO}_{2}$ max were to obtain a plateau of $\mathrm{VO}_{2}$, to reach predicted maximal values of $\mathrm{VO}_{2}$ and $\mathrm{HR}$, and to measure a respiratory quotient value higher than 1.1 [28].

\subsection{Electromyographic (EMG) Recording and Analyses}

As in our previous studies [7,26,27], bipolar (30 mm center-to-center) Ag-AgCl surface electrodes (Dantec, 13 L 20) were used to measure EMG voltage from the vastus lateralis muscle on the dominant side of the body. The electrodes were placed between the motor point and the proximal tendon. Inter-electrode impedance was kept below $2000 \mathrm{ohm}$ by careful skin shaving and abrasion with an ether pad. The EMG signal was amplified (Nihon Kohden, Tokyo, Japan; common mode rejection ratio, $90 \mathrm{~dB}$; input impedance, $100 \mathrm{mohm}$; gain, 1000 to 5000 ) with a frequency band ranging from 10 to $2000 \mathrm{~Hz}$. Compound muscle action potentials (M-waves) were evoked by direct muscle stimulation, using a monopolar technique. A constant-current neurostimulator (S88 model Grass, Quincy, MA, USA) delivered supramaximal shocks with $0.1-\mathrm{ms}$ rectangular pulses through a Grass isolation unit. One small $(1 \times 1 \mathrm{~cm})$ negative silver electrode was applied on the main motor point of the vastus lateralis muscle and a large $(3 \times 3 \mathrm{~cm})$ positive silver electrode was placed on the opposite side of the thigh. The main motor point of this muscle was identified as the location of the cathode yielding the strongest contraction with the lowest pulse amplitude. To prevent any change in the electrode impedance throughout the whole challenge, the pulse intensity was set about $15 \%$ above the level yielding an $\mathrm{M}$ wave of maximal amplitude during the $0-\mathrm{W}$ pedalling period preceding the exercise bout. The EMG signal was fed to a numerical oscilloscope (model DSO 400, Gould, Ballainvilliers, France), permitting to aver- age the M-waves from 8 successive potentials and to calculate 1) the peak M-wave amplitude; 2) its duration; and 3 ) the conduction time, that is the time between the stimulus artefact and the peak EMG response. To average $M$ waves elicited at constant muscle length, a trigger signal from a magnetic sensor coupled to the crank gear mechanism of the cycloergometer indicated the onset of the leg extension and thus of the vastus lateralis contraction. It was used to trigger within a delay of $100 \mathrm{~ms}$ the neurostimulator delivering 0.1-ms-long rectangular pulses. Because the pedalling rate was set at $60 \mathrm{~min}^{-1}$, one leg extension was performed every sec and the stimulation frequency was set at $1 \mathrm{~Hz}$. For each epoch of the protocol (the $0-\mathrm{W}$ cycling period preceding the incremental exercise, incremental exercise, and recovery period), eight successive evoked muscle potentials were averaged. Because $\mathrm{M}$ wave recordings must be performed during cycling at the same pedalling rate, the subject was asked to pedal again for several epochs during the recovery from exercise.

\subsection{Biochemical Analyses}

A catheter (Neofly 21 G, Viggo-Spectramed) was inserted in an antecubital vein. Eight $\mathrm{ml}$ of heparinized blood were sampled at different sequences of the protocol in order to measure biochemical variables. Lactic acid (LA) and potassium $\left(\mathrm{K}^{+}\right)$concentrations were measured using specific electrodes (Corning-Chiron model 860, Bayer Corporation, East Walpole, MA USA). A plasma marker of lipid peroxidation (thiobarbituric acid reactive substances, TBARS) and an antioxidant (reduced ascorbic acid, RAA) were analyzed according to the original methods by Uchiyama and Mihara [29] for TBARS and Maickel [30] for RAA. All the measurements were made in duplicate.

\subsection{Exercise Protocol}

The protocol consisted of 1) a 30-min rest period, during which all the variables were measured and venous blood samples collected; 2) a 2-min 0-W work load period; 3) a work period, and 4) a 30-min recovery period. When $\mathrm{VO}_{2}$ max was reached, venous blood was sampled and M-wave recorded. During the post-exercise recoveryperiod, venous blood samplings and $\mathrm{M}$-wave recordings were performed at 5 and $30 \mathrm{~min}$.

\subsection{Statistical Analyses}

Data are presented as means + standard error (SE). An independent Student's t-test was performed to compare the baseline levels of biochemicals between the two groups of CFS patients and control subjects. For temporally repeated data during rest, exercise, and recovery periods, 
the changes over time were determined using ANOVA when the variables were normally distributed or a Friedman's test for repeated measures when they were not. When data were normally distributed, a two-way analysis of variance for repeated measures (RM-ANOVA 2) (i.e. groups and time) was used to determine the significance of changes throughout the cycling trial and the post-exercise recovery period. Post-hoc independent ttests with Bonferroni corrections were used to compare time-point specific differences between groups. In all cases, significance was set at the two sides 0.05 level.

\section{RESULTS}

\subsection{Examination at Rest}

No intergroup differences were noted between baseline values of $\mathrm{LA}, \mathrm{K}^{+}$, and RAA concentrations whereas the mean value of resting TBARS level was significantly higher in CFS patients and almost doubled in the I CFS group (Table 1). The $\mathrm{M}$-wave characteristics measured in the vastus lateralis at rest did not significantly differ between groups (Table 2).

\subsection{Response to Incremental Maximal Cycling}

$\mathrm{VO}_{2} \max$ and maximal LA concentration did not differ between the control and CFS groups (Table 1). Figure 1 gives the kinetics of plasma TBARS, RAA, and $\mathrm{K}^{+}$levels during and after exercise in the three groups. The TBARS level culminated at $\mathrm{VO}_{2} \mathrm{max}$ in all subjects. The maximal RAA decrease was measured at $\mathrm{VO}_{2}$ max in CFS patients but later in healthy subjects, and the $\mathrm{K}^{+}$ concentration always culminated at $\mathrm{VO}_{2} \max$. Table $\mathbf{1}$ shows that compared to controls the maximal TBARS increase was almost doubled in NI CFS patients but did not significantly differ in the I CFS group. No intergroup differences were noted in the maximal RAA decrease. The peak $\mathrm{K}^{+}$increase was significantly attenuated in the I CFS group.

Marked M-wave changes occurred in CFS patients at $\mathrm{VO}_{2} \mathrm{max}$ and persisted after the exercise had ended. Figure 2 gives examples of $\mathrm{M}$-wave recordings in a control subject and a I CFS patient showing a marked depression in the latter. Figure 3 depicts the kinetics of the M-wave and CT changes in the three groups. The significance of intergroup differences was already present at $\mathrm{VO}_{2} \mathrm{max}$ and persisted at the $5^{\text {th }}$ and $30^{\text {th }} \mathrm{min}$. Table 2 shows that the largest exercise-induced M-wave variations occurred in the I CFS group. The M-wave alterations combined a decreased M-wave amplitude and a lengthened duration of both the M-wave and conduction time.

Because our CFS patients were explored 6 to 23 months after the beginning of the muscle fatigue, we searched for relationships between this delay and the magnitude of resting values and exercise-induced variations of TBARS, RAA, $\mathrm{K}^{+}$and $\mathrm{M}$-wave characteristics in NI and I CFS groups. We found that the duration of the clinical disorders did not affect the biochemical and electrophysiological variables.

\subsection{Relationships between Biochemical and Electrophysiological Variables}

As shown in Figure 4, the post-exercise changes in Mwave amplitude and CT were proportional to the baseline TBARS level, the highest alterations being measured in the I CFS group. Despite their changes were near concomitant, the maximal M-wave variations were not correlated to the maximal exercise-induced changes in TBARS, RAA, $\mathrm{K}^{+}$, and LA.

\section{DISCUSSION}

The present study confirms data previously reported in CFS patients, that is an elevation of the blood oxidant status at rest [7-13], an accentuated exercise-induced oxidative stress [7,8] and a reduced muscle excitability [7]. The new finding was the significant accentuation of the blood oxidant status disorders at rest and muscle hypoexcitability at work in CFS patients who reported a recent severe infection (I CFS group). It must be noted that the post-exercise oxidative stress (TBARS increase) was significantly enhanced in NI CFS group but not I CFS one. This might result from the marked elevation of the

Table 2. M-wave characteristics (amplitude, duration) and neuromuscular conduction time (CT) measured during pre-exercise 0 -W cycling period (reference, Ref) and their maximal exercise-induced variations in controls and the two groups of CFS patients.

\begin{tabular}{cccc}
\hline & Controls & NI CFS group & I CFS group \\
\hline M-wave amplitude Ref, mV & $47 \pm 9$ & $43 \pm 14$ & $34 \pm 16$ \\
$\Delta$ M-wave amplitude max, \% rest & $-16 \pm 6$ & $26 \pm 6^{* *}$ & $-56 \pm 10^{\# \#}$ \\
M-wave duration Ref, ms & $4.3 \pm 0.7$ & $4.2 \pm 0.9$ & $4.9 \pm 1.0$ \\
$\Delta$ M-wave duration max, \% rest & $+8 \pm 2$ & $+25 \pm 8^{\#, * *}$ & $+45 \pm 11^{\# \#}$ \\
CT Ref, ms & $0.73 \pm 0.07$ & $0.61 \pm 0.14$ & $0.83 \pm 0.15$ \\
$\Delta$ CTmax, \% rest & $+5 \pm 3$ & $+15 \pm 4^{\#,}, 7^{\# \#}$ \\
\hline
\end{tabular}

Values are mean \pm SE. Symbol ${ }^{\#}$ indicates significant difference between controls and CFS patients $\left({ }^{\#}: \mathrm{p}<0.05 ;{ }^{\# \#}: \mathrm{p}<0.01\right)$ and asterisk depicts intergroup CFS differences $\left({ }^{*}: \mathrm{p}<0.05 ;{ }^{* *}: \mathrm{p}<0.01\right)$. 

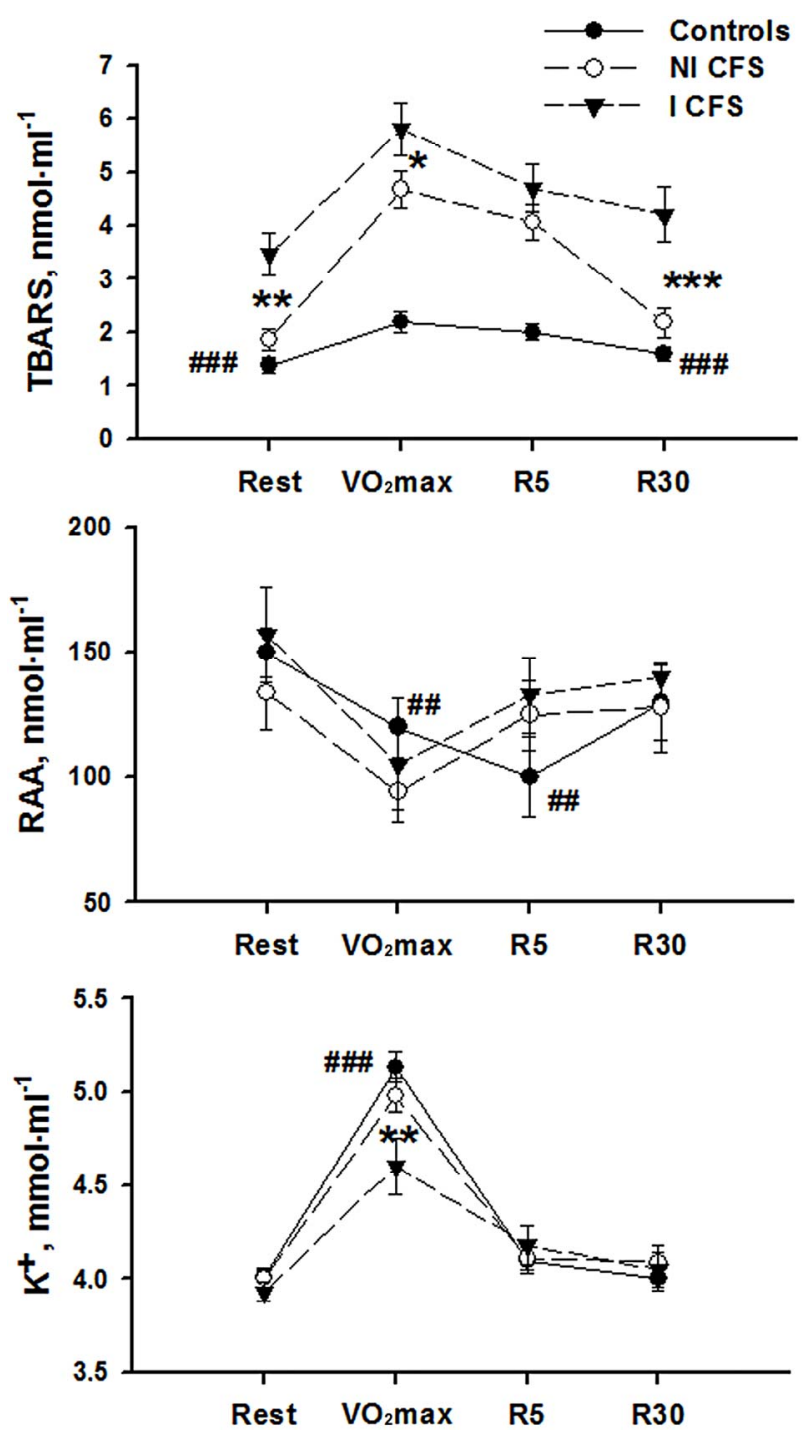

Figure 1. Plasma concentration of thiobarbituric acid reactive substances (TBARS) measured in control healthy subjects and both groups of CFS patients (non infected, or NI, and infected, or I) at rest, maximal oxygen uptake $\left(\mathrm{VO}_{2} \mathrm{max}\right)$, and during the post-exercise 30 -min recovery period. Values are mean \pm SEM. Symbol ${ }^{\#}$ denotes significant differences between controls and CFSs $\left({ }^{\#} \mathrm{p}<0.01 ;{ }^{\# \#} \mathrm{p}<0.001\right)$. Asterisk was used to depict significant differences between NI and I CFS groups ( $\left(^{*} \mathrm{p}<0.05\right.$; $\left.{ }^{* *} \mathrm{p}<0.01 ;{ }^{* * *} \mathrm{p}<0.001\right)$.

baseline TBARS concentration in I CFS patients which allows to suppose that the disorders of their oxidant status are already near maximal. The chronic elevation of the baseline TBARS level seems to play a key role in muscle excitability at work. Indeed, we measured the highest post-exercise alterations of muscle excitability in the I CFS group. We also noted a significant reduction of the muscle potassium outflow in response to maximal exercise in this group.

The strength of this study was the relative accessibility of the tools used to diagnose CFS by most of the univer-

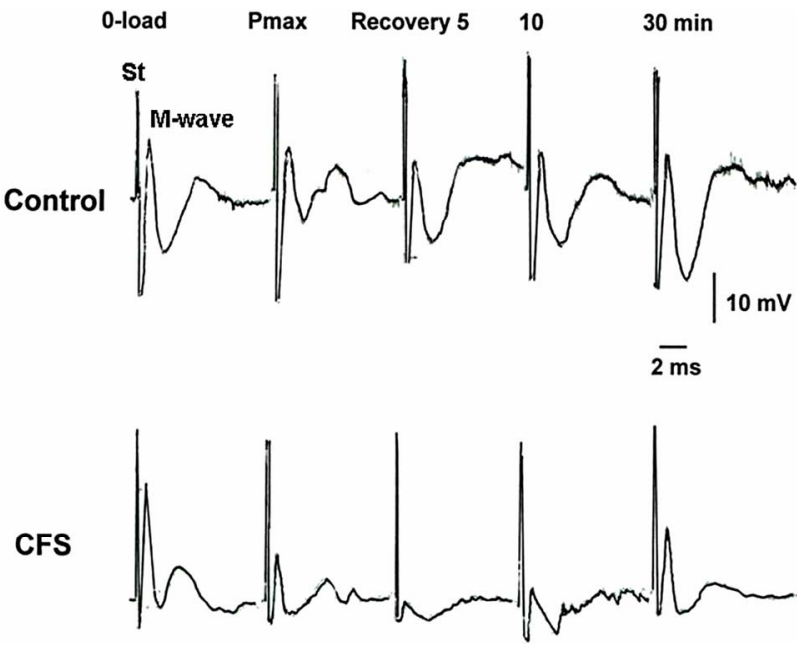

Figure 2. Examples of M-wave recordings in vastus lateralis muscle in a control (healthy) subject and a CFS patient in the I group at rest, $\mathrm{VO}_{2} \mathrm{max}$, and during the 30 -min post-exercise recovery period. Stimulus (St) and M-wave are indicated.

sity hospitals (M-wave recordings and plasma oxidantantioxidant status). Its limitation was the absence of data on values of the heat shock proteins (HSP) in our CFS groups. Indeed, our previous studies $[8,13]$ have clearly shown that both the baseline levels and exercise-induced variations of HSPs are significantly lower in these patients. These measurements were not repeated in the present study because HSP measurements with immunoassays kits are relatively expensive and can not be supported by our grants.

Our comparisons between control subjects and CFS patients at work are valuable because their $\mathrm{VO}_{2} \max$ and maximal exercise power, and thus the total duration of incremental exercise, did not significantly differ. The absence of impaired aerobic metabolism in our CFS patients corroborates several previous observations, based on the same [7] or nearly the same exercise protocol [31, 32]. The accentuated exercise-induced oxidative stress in CFS patients might partly explain the absence of any impairment of their aerobic metabolism at work. Indeed, superoxide activates the mitochondrial uncoupling proteins [33] and the uncoupling processes are well known to enhance the oxygen uptake through their influence on the mitochondrial respiratory chain.

The present study in CFS patients did not report any difference in resting values of the neuromuscular transmission (conduction time) and muscle membrane excitability compared to healthy subjects. On the other hand, we measured marked alterations of the muscle excitability at work which were already significant at the end of the incremental exercise and persisted during the recovery period. The reduction of the M-wave amplitude and the lengthening of the neuromuscular conduction time (CT) were significantly accentuated in CFS patients with 

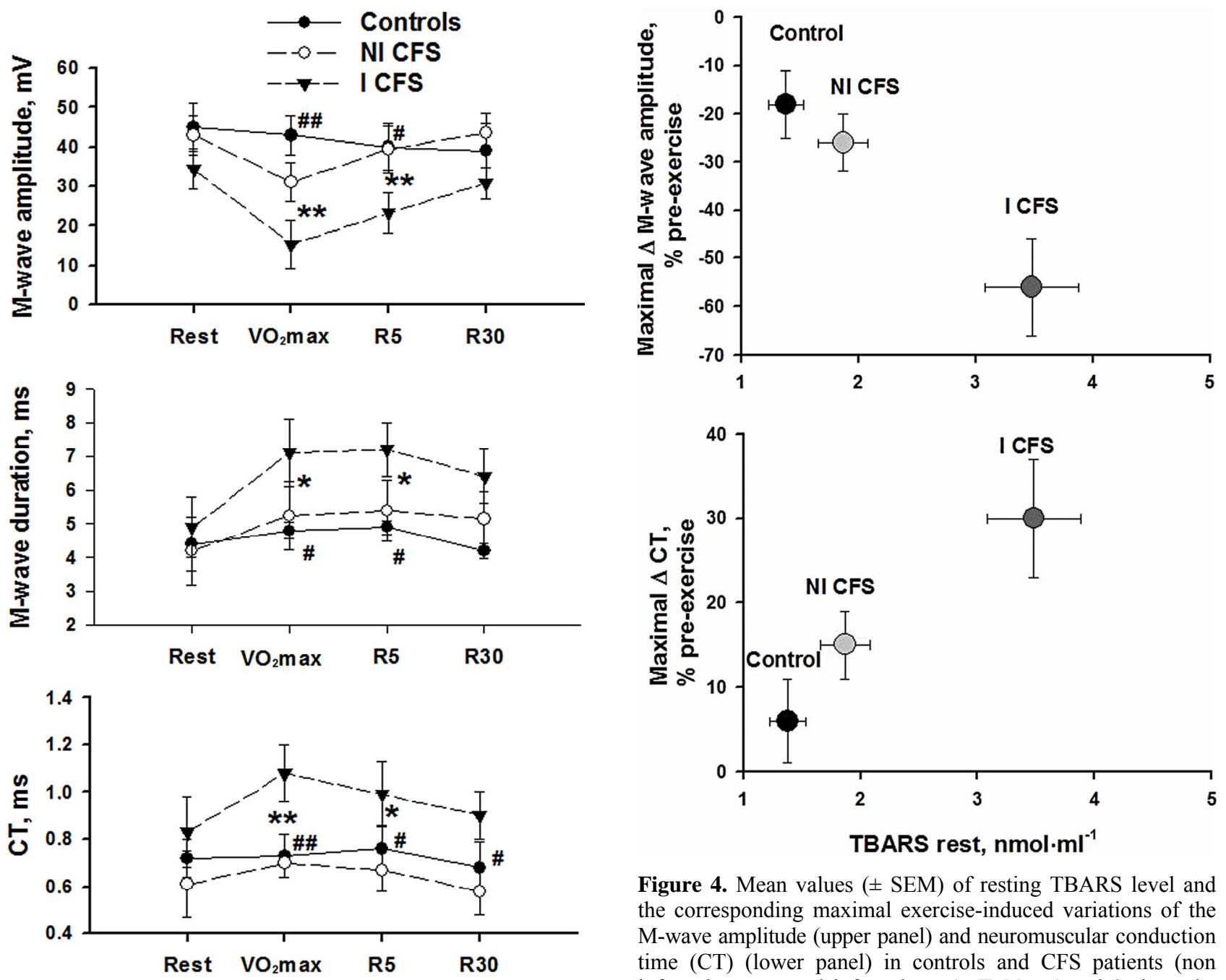

Figure 4. Mean values ( \pm SEM) of resting TBARS level and the corresponding maximal exercise-induced variations of the M-wave amplitude (upper panel) and neuromuscular conduction time (CT) (lower panel) in controls and CFS patients (non infected, or NI, and infected, or I). Tables 1 and $\mathbf{2}$ show the significance of intergroup differences.

Figure 3. Absolute values of $\mathrm{M}$-wave amplitude and duration, and the neuromuscular conduction time (CT) measured in control healthy subjects and CFS patients (non infected, or NI, and infected, or I) at rest, maximal oxygen uptake $\left(\mathrm{VO}_{2} \max \right)$ at the end of cycling exercise, and during the post-exercise 30-min recovery period. Values are mean \pm SEM. Symbol ${ }^{\#}$ denotes significant differences between controls and CFS groups $\left({ }^{\#} \mathrm{p}<\right.$ $\left.0.05 ;{ }^{\#} \mathrm{p}<0.01\right)$. Asterisk was used to depict significant differences between NI and I CFS groups $\left({ }^{*} \mathrm{p}<0.05 ;{ }^{* *} \mathrm{p}<\right.$ $0.01)$.

history of recent severe infection. The enhanced alterations of post-exercise muscle membrane excitability in our I CFS group were associated with a significant reduction of the maximal potassium outflow measured at the end of exercise. We already reported literature data [15-18] demonstrating close relationships between the oxidative stress, the $\mathrm{K}^{+}$exchanges through the sarcolemma, and the muscle excitability. As demonstrated by Marcos and Ribas [18], an extracellular potassium accumulation can act as a negative feedback signal for sarcolemma excitability and might constitute a possible mechanism for the post-exercise M-wave alterations.

The present study indicates that CFS patients with antecedents of severe infection have higher biological and EMG disorders than other patients who solely reported practice of high physical activity before the occurrence of fatigue symptoms. It is well documented that acute infection constitutes a stress responsible for an oxidative stress [21-24]. Our major observation was that infection was responsible for lengthened alterations of the resting oxidant status. Indeed, the episode of infection occurred within the three to seven month period preceding the onset of CFS symptoms and our CFS patients were explored 6 to 23 months after the beginning of fatigue. A persistent enhanced oxidant production is highly suspected to trigger the production of the tumor necrosis factor alpha, the most clearly defined mediator of contractile dysfunction. Some studies failed to demonstrate abnormal plasma cytokine levels in CFS patients at rest compared to healthy subjects $[34,35]$ while others reported an elevation of plasma level of transforming growth fac- 
tor-beta level, IL-6, and Il- $1 \alpha[36,37]$.

In conclusion, we verified our hypothesis that severe infection represents a stressor responsible for a lengthened alteration of blood oxidant status which could explain the impaired exercise-induced muscle potassium outflow and the altered membrane excitability in CFS patients. In these patients, free radical damage may be a contributor to the underlying functional defects during exercise and symptom presentation.

\section{REFERENCES}

[1] Holgate, S.T., Komaroff, A.L., Mangan, D. and Wessely, S. (2011) Viewpoint: Chronic FAtigue syndrome: Understanding a complex illness. Nature Reviews Neuroscience, 12, 539-544. doi:10.1038/nrn3087

[2] Prins, J.B., Van der Meer, J.W.M. and Bleijenberg, G. (2006) Chronic fatigue syndrome. Lancet, 367, 346-355. doi:10.1016/S0140-6736(06)68073-2

[3] Goodwin, L., White, P.D., Hotopf, M., Stansfeld, S.A. and Clark, C. (2011) Psychopathology and physical activity as predictors of chronic fatigue syndrome in the 1958 british birth cohort: A replication study of the 1946 and 1970 birth cohorts. Annals of Epidemiology, 21, 343350. doi:10.1016/j.annepidem.2010.12.003

[4] Bassi, N., Amital, D., Amital, H., Doria, A. and Shoenfeld, Y. (2008) Chronic fatigue syndrome: characteristics and possible causes for its pathogenesis. The Israel Medical Association Journal, 10, 79-82.

[5] Evengard, B., Jonzon, E., Sandberg, A., Theorell, T. and Lindh, G. (2003) Differences between patients with chronic fatigue syndrome and with chronic fatigue at an infectious disease clinic in Stockolm, Sweden. Psychiatry and Clinical Neurosciences, 57, 361-368. doi:10.1046/j.1440-1819.2003.01132.x

[6] Naess, H., Sundal, E., Myhr, K.M. and Nyland, H.I. (2010) Postinfectious and chronic fatigue syndromes: Clinical experience from a tertiary-referral centre in Norway. In Vivo, 24, 185-188.

[7] Jammes, Y., Steinberg, J.G., Mambrini, O., Bregeon, F. and Delliaux, S. (2005) Chronic fatigue syndrome: Assessment of increased oxidative stress and altered muscle excitability in response to incremental exercise. Journal of Internal Medicine, 257, 299-310. doi:10.1111/j.1365-2796.2005.01452.x

[8] Jammes, Y., Steinberg, J.G., Delliaux, S. and Bregeon, F. (2009) Chronic fatigue syndrome combines increased exercise-induced oxidative stress and reduced cytokine and Hsp responses. Journal of Internal Medicine, 266, 196206. doi:10.1111/j.1365-2796.2009.02079.x

[9] Manuel y Keenoy, B., Moorkens, G., Vertommen, J. and De Leeuw, I. (2001) Antioxidant status and lipoprotein peroxidation in chronic fatigue syndrome. Life Sciences, 68, 2037-2049. doi:10.1016/S0024-3205(01)01001-3

[10] Vecchiet, J., Cipollone, F., Falasca, K., Mezzetti, A., Pizzigallo, E., Bucciarelli, T., De Laurentis, S., Affaitati, G., De Cesare, D. and Giamberardino, M.A. (2003) Relationship between musculoskeletal symptoms and blood markers of oxidative stress in patients with chronic fatigue syndrome. Neuroscience Letters, 335, 151-154. doi:10.1016/S0304-3940(02)01058-3

[11] Kennedy, G., Spence, V.A., McLaren, M., Hill, A., Underwood, C. and Belch, J.J. (2005) Oxidative stress levels are raised in chronic fatigue syndrome and are associated with clinical symptoms. Free Radical Biology \& Medicine, 39, 584-589. doi:10.1016/j.freeradbiomed.2005.04.020

[12] Richards, R.S., Roberts, T.K., McGregor, N.R., Dunstan, R.H. and Butt, H.L. (2000) Blood parameters indicative of oxidative stress are associated with symptom expression in chronic fatigue syndrome. Redox Report, 5, 35-41.

[13] Jammes, Y., Steinberg, J.G. and Delliaux, S. (2012) Chronic fatigue syndrome: Acute infection and history of physical activity affect resting levels and response to exercise of plasma oxidant/antioxidant status and heat shock proteins. Journal of Internal Medicine, 272, 74-84. doi:10.1111/j.1365-2796.2011.02488.x

[14] Juel, C. (2006) Muscle fatigue and reactive oxygen species. The Journal of Physiology, 576, 279-288.

[15] Jabr, R.I. and Cole, W.C. (1993) Alterations in electrical activity and membrane currents induced by intracellular oxygen-derived free radical stress in guinea pig ventricular myocytes. Circulation Research, 72, 1229-1244. doi:10.1161/01.RES.72.6.1229

[16] Luin, E., Giniatullin, R. and Sciancalepore, M. (2011) Effects of $\mathrm{H}_{2} \mathrm{O}_{2}$ on electrical membrane properties of skeletal myotubes. Free Radical Biology \& Medicine, 50, 337 344. doi:10.1016/i.freeradbiomed.2010.11.015

[17] Sjøgaard, G. (1990) Exercise-induced muscle fatigue: The significance of potassium. Acta Physiologica Scandinavica. Supplementum, 593, 1-63.

[18] Marcos, E. and Ribas, J. (1995) Kinetics of plasma potassium concentrations during exhausting exercise in trained and untrained men. European Journal of Applied Physiology, 71, 207-214. doi:10.1007/BF00854980

[19] Fulle, S., Belia, S., Vecchiet, J., Morabito, C., Vecchiet, L. and Fano, G. (2003) Modification of the functional capacity of sarcoplasmic reticulum membranes in patients suffering from chronic fatigue syndrome. Neuromuscular Disorders, 13, 479-484. doi:10.1016/S0960-8966(03)00042-7

[20] Chakraborty, S.P., Das, S., Chattopadhyay, S., Tripathy, S., Dash, S.K., Pramanik, P. and Roy, S. (2012) Staphylococcus aureus infection induced redox signalling and DNA fragmentation in T-lymphocytes: possible ameliorative role of nanoconjugated vancomycin. Toxicology Mechanisms and Methods, 22, 193-204. doi:10.3109/15376516.2011.629236

[21] Biagoli, M.C., Kaul, P., Singh, I. and Turner, R.B. (1999) The role of oxidative stress in rhinovirus induced elaboration of IL-8 by respiratory epithelial cells. Free Radical Biology \& Medicine, 26, 454-462. doi:10.1016/S0891-5849(98)00233-0

[22] Choi, A.M., Knobil, K., Otterbein, S.L., Eastman, D.A. and Jacoby, D.B. (1996) Oxidant stress responses in influenza virus pneumonia: Gene expression and transcription factor activation. American Journal of Physiology, 
271, L383-L391.

[23] Hosakote, Y.M., Liu, T., Castro, S.M., Garofalo, R.P. and Casola, A. (2009) Respiratory syncitial virus induces oxidative stress by modulating antioxidant status. American Journal of Respiratory Cell and Molecular Biology, 41, 348-357. doi:10.1165/rcmb.2008-03300C

[24] Al-Nimer, M.S., Mahmood, M.M. and Khazaal, S.S. (2011) Nitrostative stress status during seasonal and pdmH1N1 infection in Iraq. The Journal of Infection in Developing Countries, 5, 863-867. doi:10.3855/jidc.1505

[25] Yamada, Y., Limmon, G.V., Zheng, D., Li, N., Li, L., Yin, L., Chow, V.T., Chen, J. and Engelward, B.P. (2012) Major shifts in the spatio-temporal distribution of lung antioxidant enzymes during influenza pneumonia. PLOS One, 7, ArticleID: e31494. doi:10.1371/journal.pone.0031494

[26] Arnaud, S., Zattara-Hartmann, M.C., Tomei, C. and Jammes, Y. (1997) Correlation between muscle metabolism and changes in M-wave and surface electromyogram: Dynamic constant load leg exercise in untrained subjects. Muscle \& Nerve, 20, 1197-1199. doi:10.1002/(SICI)1097-4598(199709)20:9<1197::AIDMUS20>3.0.CO;2-P

[27] Jammes, Y., Zattara-Hartmann, M.C., Caquelard, F., Arnaud, S. and Tomei, C. (1997) Electromyographic changes in vastus lateralis during dynamic exercise. Muscle \& Nerve, 20, 247-249. doi:10.1002/(SICI)1097-4598(199702)20:2<247::AID-M US21>3.0.CO;2-Z

[28] Wasserman, K. (1987) Determinants and detection of anaerobic threshold and consequences of exercise above it. Circulation, 76, V129-V139.

[29] Uchiyama, M. and Mihara, M. (1978) Determination of malonedialdehyde precursor in tissues by thiobarbituric acid test. Analytical Biochemistry, 86, 271-278. doi:10.1016/0003-2697(78)90342-1

[30] Maickel, R.P. (1960) A rapid procedure for the determination of adrenal ascorbic acid. Application of the Sullivan and Clarke method to tissues. Analytical Biochemistry, 1, 498-501. doi:10.1016/0003-2697(60)90063-4
[31] Mullis, R., Campbell, I.T., Wearden, A.J., Morriss, R.K. and Pearson, D.J. (1999) Prediction of peak oxygen uptake in chronic fatigue syndrome. British Journal of Sports Medicine, 33, 352-356. doi:10.1136/bjsm.33.5.352

[32] Sargent, C., Scroop, G.C., Nemeth, P.M., Burnet, R.B. and Buckle, J.D. (2002) Maximal oxygen uptake and lactate metabolism are normal in chronic fatigue syndrome. Medicine \& Science in Sports \& Exercise, 34, 51-56. doi:10.1097/00005768-200201000-00009

[33] Echtay, K.S., Roussel, D., St-Pierre, J., Jekabsons, M.B., Cadenas, S., Stuart, J.A., Harper, J.A., Roebuck, S.J., Morrison, A., Pickering, S., Clapham, J.C. and Brand, M.D. (2002) Superoxide activates mitochondrial uncoupling proteins. Nature, 415, 96-99. doi:10.1038/415096a

[34] Amel Kashipaz, M.R., Swinden, D., Todd, I. and Powell, R.J. (2003) Normal production of inflammatory cytokines in chronic fatigue and fibromyalgia syndromes determined by intracellular cytokine staining in short-term cultured blood mononuclear cells. Clinical \& Experimental Immunology, 132, 360-365. doi:10.1046/j.1365-2249.2003.02149.x

[35] Vollmer-Conna, U., Cameron, B., Hadzi-Pavlovic, D., Singletary, K., Davenport, T., Vernon, S., Reeves, W.C., Hickie, I., Wakefield, D., Lloyd, A.R. (2007) Dubbo Infective Outcomes Study Group. Postinfective fatigue syndrome is not associated with altered cytokine production. Clinical Infectious Diseases, 45, 732-735. doi:10.1086/520990

[36] Gaab, J., Rohleder, N., Heitz, V., Engert, V., Schad, T., Schürmeyer, T.H. and Ehlert, U. (2005) Stress-induced changes in LPS-induced pro-inflammatory cytokine production in chronic fatigue syndrome. Pychoneuroendocrinology, 30, 188-198.

[37] Chao, C.C., Janoff, E.N., Hu, S.X., Thoma, K., Gallagher, M., Tsang, M. and Peterson, P.K. (1991) Altered cytokine release in peripheral blood mononuclear cell cultures from patients with the chronic fatigue syndrome. Cytokine, 3, 292-298. doi:10.1016/1043-4666(91)90497-2 